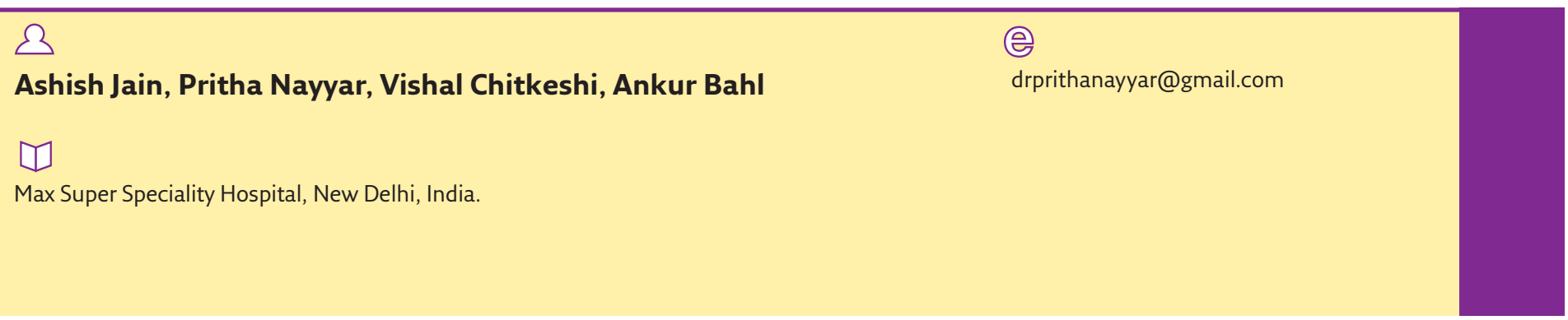

\title{
An unusual complication after endobronchial ultrasound-guided transbronchial needle aspiration
}

\section{Case report}

Bronchoscopy with endobronchial ultrasound-guided transbronchial needle aspiration (EBUS-TBNA) is considered to be a safe procedure and is far less invasive than mediastinoscopy or percutaneous needle biopsy. It has been increasingly utilised for the diagnosis of sarcoidosis and unexplained mediastinal adenopathy, and for lung cancer staging. Complications from EBUS-TBNA are rare. Complications that have been reported are haemorrhages, pneumothorax, infectious complications like mediastinitis, pleuropericarditis, abscess, respiratory failure, airway injury and hypotension. Some of the patients also require intervention like tube drainage for pneumothorax [1].

An 86-year-old male, current smoker presented with complaints of shortness of breath for 2 years followed by cough with minimum expectoration and chest pain for 5 months. Contrast-enhanced computed tomography of the chest showed a left lower lobe apical segment mass lesion with mediastinal lymphadenopathy with left side effusion and bilateral emphysematous changes.

Positron emission tomography/computed tomography scans (figure 1) showed a 2-fluoro-2deoxy-D-glucose (FDG)-avid (maximum standardised uptake value of 11) heterogeneously enhancing mediastinal pleural based, soft tissue density mass lesion with areas of cavitation noted in the left lower lobe, measuring $6 \times 4.4 \mathrm{~cm}$ and encasing the segmental branches of the left lower lobe bronchus. It was closely abutting the pulmonary artery and left inferior pulmonary vein. Faintly FDG-avid and nonavid, subcentimetre-sized pretracheal, precarinal, subcarinal, aortopulmonary window and left tracheobronchial lymph nodes were seen.
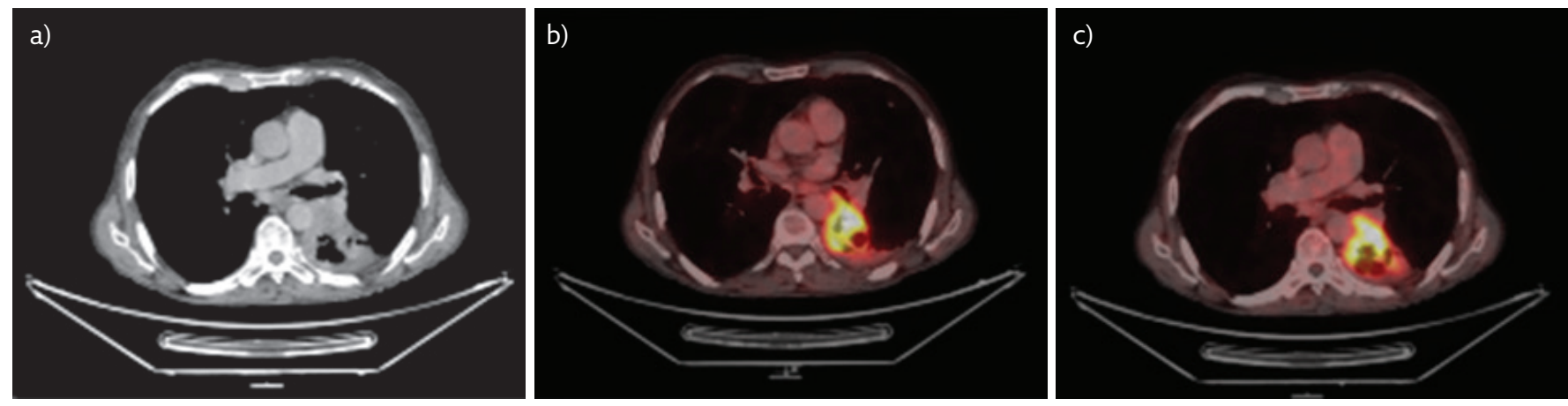

Figure 1 Positron emission tomography/computed tomography scans showed a mass lesion. a) Areas of cavitation were noted in the left lower lobe, encasing the segmental branches of the left lower lobe bronchus. b and c) FDG imaging showed an FDG-avid cavitating mass lesion in the left lower lobe, encasing the segmental branches of the left lower lobe bronchus.

Cite as: Jain A, Nayyar P, Chitkeshi V, et al. An unusual complication after endobronchial ultrasoundguided transbronchial needle aspiration. Breathe 2018; 14: e6-e11. 


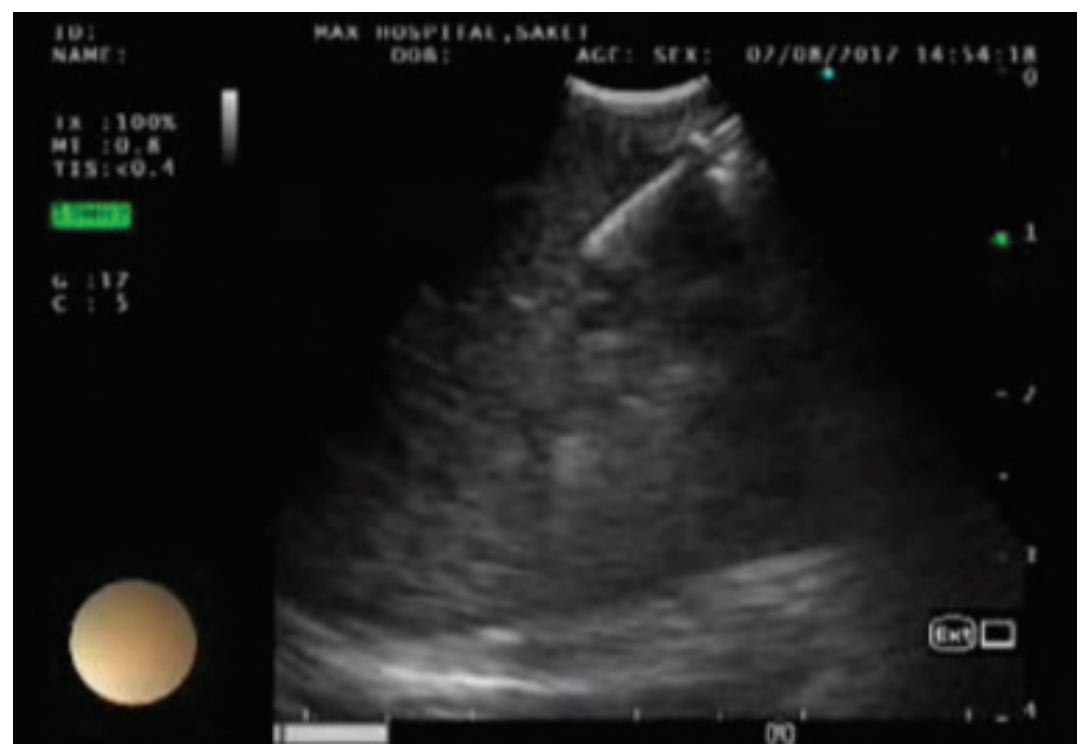

Figure 2 EBUS-TBNA from the left lower lobe mass.
Fibreoptic bronchoscopy was performed, which was normal with no endobronchial growth. EBUS-TBNA was performed from nodal stations 4R (lower right paratracheal lymph node), 7 (subcarinal lymph node), 10R and 10L (right and left hilar lymph nodes), and from the left lower lobe mass lesion (figure 2). Rapid on-site evaluation was done to check for adequacy of samples. An aspirate was obtained from the left lower lobe mass lesion using sustained suction by syringe during the puncture by the TBNA needle. This sample was sent as a cell block after squirting the aspirate on filter paper and then placing it into $10 \%$ formalin for subsequent processing in the laboratory. The final histopathology was suggestive of nonsmall cell carcinoma. Among the lymph node stations that were sampled, the subcarinal lymph node was positive for malignant cells and rest were suggestive of reactive lymph nodes.

Task 1

Which lymph node station is not easily accessible with EBUS?
a. 4
b. 7
c. $10 \mathrm{R}, 10 \mathrm{~L}$
d. 8 


\section{Answer 1}

d. Lymph node station 8 (para-oesophageal) is best accessed by endoscopic ultrasound, not EBUS.

Fibreoptic bronchoscopy and EBUS were performed by the nasal route under conscious sedation using $2 \mathrm{mg}$ midazolam and $50 \mu \mathrm{g}$ fentanyl. Heart rate, arterial oxygen saturation measured by pulse oximetry $\left(\mathrm{SpO}_{2}\right)$ and blood pressure were monitored throughout the procedure and subsequently for $2 \mathrm{~h}$. The procedure was uneventful and the patient tolerated it well. He was comfortable without any significant bout of coughing.

Next day, the patient reported swelling in the face, neck and chest, with vague chest pain. The chest pain was continuous dull aching, on the left side anteriorly radiating to the back, left arm and right side of chest, aggravated upon coughing and deep inspiration. There was no haemodynamic or respiratory compromise. On physical examination, the patient had tachycardia with a pulse rate of 120 beats $\mathrm{min}^{-1}, \mathrm{SpO}_{2}$ of $95 \%$ on room air, blood pressure of $118 / 70 \mathrm{mmHg}$ and no tachypnoea. On palpation, crepitus was present along the entire left side of the chest anteriorly and posteriorly, up to the neck and face below the level of the nasal bridge. It extended to the right side of the chest across the midline. On systemic examination, bilateral air entry was present in the chest on auscultation. Heart sounds were regular with no murmur. The rest of the examination was unremarkable. An ECG was done and was normal. Cardiac enzymes were normal. Laboratory examinations were normal.

Task 2

What complication of EBUS-TBNA has probably occurred?

a. Pneumothorax and pneumomediastinum

b. Respiratory failure

c. Mediastinitis

d. Pneumomediastinum and subcutaneous emphysema 


\section{Answer 2}

$\mathrm{d}$. The patient has developed

pneumomediastinum with subcutaneous emphysema. Pneumomediastinum represents extraluminal gas in the mediastinum. It occurs due to increased intrathoracic pressure causing alveolar rupture followed by air dissection through the bronchovascular sheath into the mediastinum. Air can also dissect through other serous structures and subcutaneous tissue. It is associated with a triad of thoracic pain, subcutaneous emphysema and dyspnoea. The most common finding is subcutaneous emphysema. Hamman's sign (detected in $10-20 \%$ of cases), characterised by systolic crackles in the left sternal border, best heard in left lateral decubitus, is considered pathognomonic [2]. In our case, the patient developed crepitus with swelling and retrosternal chest pain, which directly indicates subcutaneous emphysema with pneumomediastinum. On respiratory examination, air entry was equal bilaterally, which rules out pneumothorax.

Chest radiography showed pneumomediastinum and subcutaneous emphysema with no pneumothorax (figure 3).

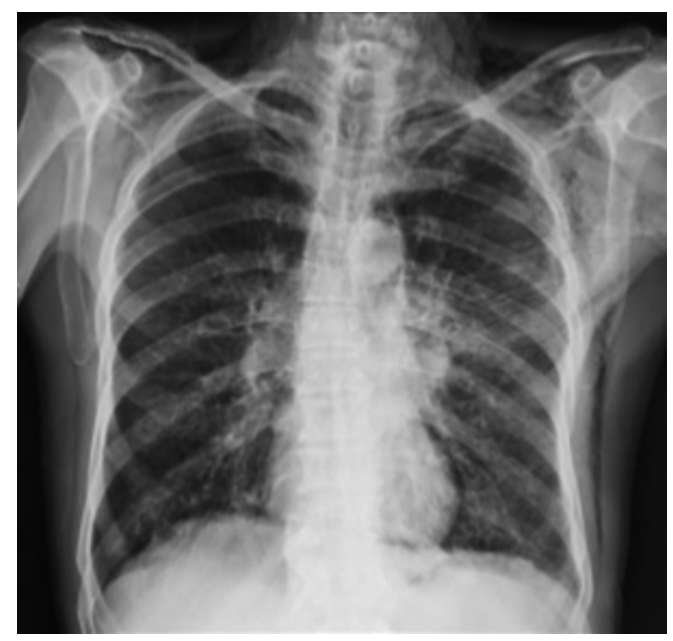

Figure 3 Chest radiograph suggestive of pneumomediastinum (air outlining the mediastinal structures) and subcutaneous emphysema in the area of the left axilla, with a continuous diaphragm sign and a ring-around-the-artery sign seen

\section{Task 3}

Which of the following is not a radiological sign of pneumomediastinum?
a. Naclerio's $V$ sign
b. Deep sulcus sign
c. Continuous diaphragm sign
d. Thymic sail sign 


\section{Answer 3}

b. The deep sulcus sign is seen in pneumothorax in supine chest radiographs when air in the pleural cavity collects in the anterolateral pleural space, outlining the deep costophrenic angle. There are several radiographic signs of pneumomediastinum, such as pneumopericardium, continuous diaphragm sign, continuous left hemidiaphragm sign, Naclerio's V sign, $V$ sign at confluence of brachiocephalic veins, ringaround-the-artery sign, thymic spinnaker-sail sign, and extrapleural air sign [3].

The patient was managed conservatively with highflow oxygen, analgesics and cough suppressants. The subcutaneous emphysema resolved gradually and the patient was discharged after 4 days. On follow-up in the outpatients' department after 7 days, there was complete resolution of swelling and chest pain. Chest radiography performed at that time showed complete resolution of the subcutaneous emphysema (figure 4).

\section{Discussion}

EBUS-TBNA is a safe procedure and complications are rare. In a nationwide survey in Japan, EBUSTBNA was performed in 7345 cases in 210 facilities (46.2\% of the facilities that responded) using a convex probe ultrasound bronchoscope, for mediastinal and hilar lesions and lung parenchymal lesions. The complication rate was $1.23 \%$, with haemorrhage being the most frequent complication $(0.68 \%)$, followed by infectious complications (0.19\%) (mediastinitis $n=7$, pneumonia $n=4$, pericarditis $n=1$, cyst infection $n=1$, and sepsis $n=1$ ). Pneumothorax developed in two cases $(0.03 \%)$, one of which required tube drainage. Prolonged hospitalisation was observed in 14 cases, lifethreatening conditions in four, and death in one (mortality rate $0.01 \%$ ) [1].

In the American College of Chest Physicians Quality Improvement Registry, Evaluation, and Education (AQuIRE) database there were no cases of pneumomediastinum or subcutaneous emphysema reported after transbronchial lung biopsy and radial EBUS-guided TBNA [4]. In a meta-analysis by voN BARTHELD et al. [5] on complications after endosonographic evaluation of mediastinum including 16,181 patients, 23 serious adverse events (0.14\%) and 35 minor adverse events $(0.22 \%)$ were observed, out of which only two pneumothoraces were reported, both requiring chest tube drainage. In our case, the patient developed pneumomediastinum and subcutaneous emphysema without pneumothorax and did not require any intervention. In our centre, out of 2000 EBUS-guided TBNA procedures performed to date, no pneumomediastinum had been reported previously. There are only a few case

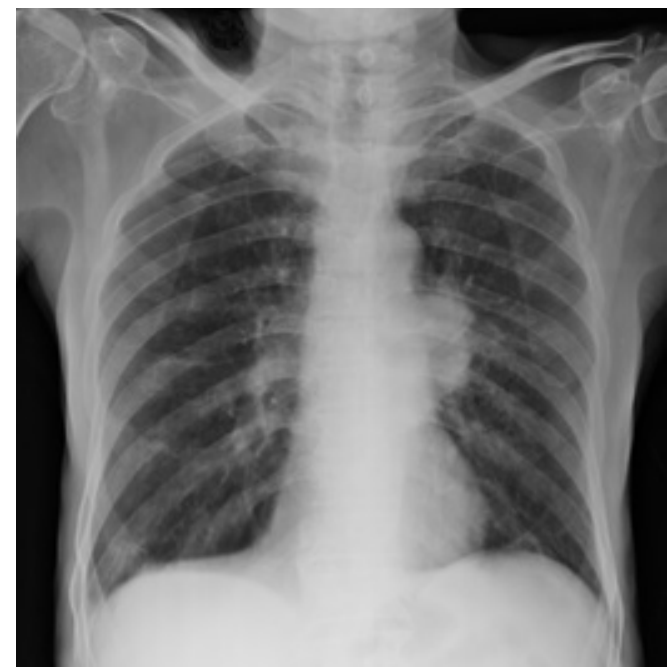

Figure 4 Follow-up normal chest radiograph with resolution of the subcutaneous emphysema and pneumomediastinum.

reports of pneumomediastinum associated with EBUS-TBNA reported so far [6-8].

The most common causes of pneumomediastinum are soft tissue infection, mucosal disruption and spontaneous pneumomediastinum. Most cases of iatrogenic pneumomediastinum are caused by the Macklin effect or ventilation-induced barotrauma, which causes mucosal breaching and spreading of air to nearby tissues via the fascial planes that connect them $[9,10]$. However, in our case, bronchoscopy was performed without any positive pressure support. In our case, the cause of pneumomediastinum and subcutaneous emphysema could be blunt airway trauma by the bronchoscope or TBNA needle. In addition, forceful coughing may cause tracheobronchial injury and increase intra-alveolar pressure, which can cause pneumomediastinum [3].

Treatment should be aimed at decreasing intrathoracic pressure by reducing coughing and straining. Oxygen therapy may hasten reabsorption of the subcutaneous nitrogen bubble as it does for a pneumothorax [11]. Since our patient was haemodynamically stable and did not have a pneumothorax, he was managed conservatively and required no intervention.

Our case report highlights this rare complication and alerts pulmonologists to the importance of early recognition, which can prevent life-threatening situations.

\section{Conclusion}

Although EBUS-TBNA is considered a safe procedure with rare complications, it is necessary to remember these severe life-threatening complications. Therefore, post-procedure detailed evaluation of patients to rule out any complication can reduce the morbidity associated with them. 


\section{Conflict of interest}

None declared.

\section{References}

1. Asano F, Aoe M, Ohsaki Y, et al. Complications associated with endobronchial ultrasound-guided transbronchial needle aspiration: a nationwide survey by the Japan Society for Respiratory Endoscopy. Respir Res 2013; 14: 50.

2. Meireles J, Neves S, Castro A, et al. Spontaneous pneumomediastinum revisited. Respir Med CME 2011; 4: 181-183.

3. Bejvan SM, Godwin JD. Pneumomediastinum: old signs and new signs. AJR Am / Roentgenol 1996; 166: 1041-1048.

4. Eapen GA, Shah AM, Lei X, et al. Complications, consequences, and practice patterns of endobronchial ultrasound-guided transbronchial needle aspiration: results of the AQuIRE registry. Chest 2013; 143: 1044-1053.

5. von Bartheld MB, van Breda A, Annema JT. Complication rate of endosonography (endobronchial and endoscopic ultrasound): a systematic review. Respiration 2014; 87: 343-351.

6. Ortiz R, Hayes M, Arias S, et al. Pneumomediastinum and pneumopericardium after endobronchial ultrasound-guided transbronchial needle aspiration. Ann Am Thor Soc 2014; 11 680-681.

7. Liang JJ, Midthun DE. Postbronchoscopy pneumomediastinum and subcutaneous emphysema. Intern Med 2013; 52: 519-520.

8. Shweihat Y, Perry JD, Munn N. Severe pneumomediastinum complicating EBUS-TBNA. J Bronchol Intervent Pulmonol 2015 22: e8-e9.

9. Macklin CC. Transport of air along sheaths of pulmonic blood vessels from alveoli to mediastinum. Arch Intern Med 1939 64: 913-926.

10. Kumar A, Pontoppidan H, Falke KJ, et al. Pulmonary barotrauma during mechanical ventilation. Crit Care Med 1973; 1: 181-186.

11. Maunder RJ, Pierson DJ, Hudson LD. Subcutaneous and mediastinal emphysema: pathophysiology, diagnosis, and management. Arch Intern Med 1984; 144: 1447-1453. 